\title{
Cognitive impairment and hypertension among Chinese nonagenarians and centenarians
}

\author{
Chang-Quan Huang ${ }^{1,2}$, Bi-Rong Dong ${ }^{1}$, Yan-Ling Zhang ${ }^{1}$, Hong-Mei Wu ${ }^{1}$, Qing-Xiu Liu ${ }^{2}$ and Joseph H Flaherty ${ }^{3}$
}

In this study, we explore the association between hypertension and cognitive impairment in the very elderly, using a sample ranging in age from 90 to 108 years. This work was conducted as a cross-sectional study. Our population included 782 unrelated Chinese nonagenarians and centenarians (67.5\% women, mean age 93.62 years). The mean cognitive function score for the sample was 14.95 (s.d.: 5.99, range: 0-28). There were no significant differences between individuals with and without hypertension with regard to cognitive function scores $(14.95 \pm 6.01$ vs. $14.95 \pm 5.82, P=0.997)$ or cognitive impairment prevalence (59.52 vs. 59.42, $P=0.976)$. There were also no significant differences in the prevalence of hypertension $(56.99$ vs. $57.10, P=0.976)$ or in the levels of arterial blood pressure (including systolic blood pressure (SBP) and diastolic blood pressure (DBP)) (139.86 \pm 22.69 vs. $140.28 \pm 23.51, P=0.799$ and $73.05 \pm 12.07$ vs. $72.11 \pm 12.06, P=0.678$, for SBP and DBP, respectively) between individuals with and without cognitive impairment. Multiple logistic regression showed that cognitive impairment and hypertension were not risk factors for each other (odds ratio (OR) of cognitive impairment as a function of increased hypertension: 0.938 $(0.655,1.341)$; OR of hypertension as a function of increased cognitive impairment: $0.920(0.643,1.317))$. In summary, we found that cognitive impairment was not directly correlated with hypertension among Chinese nonagenarians and centenarians. Hypertension Research (2009) 32, 554-558; doi:10.1038/hr.2009.72; published online 29 May 2009

Keywords: Chinese; cognitive impairment; hypertensive; nonagenarians and centenarians

\section{INTRODUCTION}

About $10 \%$ of people older than 65 years have cognitive impairment, ranging from mild deficits to dementia. ${ }^{1}$ Hypertension has been viewed as a common risk factor for cognitive disorders. ${ }^{2-4}$ Many prospective and cross-sectional observational studies have shown that hypertension is associated with cognitive decline in older persons. ${ }^{5-14}$ Common to each of these studies, however, has been the relatively young age of the participants. The average age of these populations has been under 85 years; only a few studies have included individuals aged 90 years or above, and no studies have focused only on individuals aged 90 years or older. Cognitive impairment and hypertension are both major components of age-related deterioration. ${ }^{15,16}$ As the population of older persons increases, the number of cognitively impaired older individuals is expected to rise. ${ }^{17,18}$ To the best of our knowledge, no studies have explored whether cognitive impairment is associated with hypertension among very old people, that is, those aged 90 years and above. In this study, we sought to observe the association of cognitive impairment with hypertension among very old people using a cohort of Chinese men and women aged 90-108 years.

\section{METHODS}

Study participants

The methods of this study have been reported previously. ${ }^{19}$ In brief, as part of the Project of Longevity and Aging in Dujiangyan, a cross-sectional study of age-related diseases was conducted in 870 long-living individuals ( $>90$ years) on the basis of the Dujiangyan (located in Sichuan province, southwest China) 2005 census. The aims of the Project of Longevity and Aging in Dujiangyan were to investigate the relationship between environment, lifestyle, genetics, longevity and age-related diseases. Participants were examined by trained professional physicians using basic health criteria, and age-related diseases were recorded, including cancer, type 2 diabetes, hypertension, heart failure, chronic obstructive pulmonary disease, Alzheimer's and Parkinson's diseases and so on. In this analysis, long-living individuals with cancer, secondary hypertension, severe heart failure and terminal stage of chronic obstructive pulmonary disease were excluded. Our study population ultimately consisted of 782 long-living individuals. Informed consent was obtained from all participants (or their legal proxies). The Research Ethics Committee of the Sichuan University approved the study.

\section{Data collection and measurements}

Measurement of blood pressure. Right-arm blood pressure (BP) was measured with participants in the sitting or recumbent position by trained nurses or physicians. BP was taken twice, to the nearest $2 \mathrm{~mm} \mathrm{Hg}$, using a standard mercury sphygmomanometer (phases I and $\mathrm{V}$ of Korotkoff). The mean value of the two measurements was used to calculate systolic BP (SBP) and diastolic BP (DBP), and the SBP and DBP were calculated as the mean of the right- and left-arm values in exceptional cases (upper-extremity vascular disease). The mean of the two readings was used for the classification of BP according to the Joint National Committee VII criteria into normal $(\mathrm{SBP}<120 \mathrm{~mm} \mathrm{Hg}$ and $\mathrm{DBP}<80 \mathrm{~mm} \mathrm{Hg}$ ), pre-hypertension (SBP $120-139 \mathrm{~mm} \mathrm{Hg}$ and/or DBP

1Department of Geriatrics, West China Hospital, Sichuan University, Sichuang province, China; ${ }^{2}$ Department of Geriatrics, The Third Hospital of Mianyang, Mianyang, Sichuan, China and ${ }^{3}$ Geriatric Research, Education and Clinical Center, St Louis Veteran's Affairs Medical Center, St Louis, MO, USA 
$80-89 \mathrm{~mm} \mathrm{Hg}$ ), stage 1 hypertension (SBP $140-159 \mathrm{~mm} \mathrm{Hg}$ and/or DBP $90-99 \mathrm{~mm} \mathrm{Hg}$ ) or stage 2 hypertension (SBP $>160 \mathrm{~mm} \mathrm{Hg}$ and/or DBP $>$ $100 \mathrm{~mm} \mathrm{Hg}$ ). Isolated systolic hypertension was defined as SBP $>140 \mathrm{~mm} \mathrm{Hg}$ and $\mathrm{DBP}<90 \mathrm{~mm} \mathrm{Hg}$, and isolated diastolic hypertension was defined as $\mathrm{SBP}<140 \mathrm{mmHg}$ and $\mathrm{DBP}>90 \mathrm{mmHg}$. Hypertension was defined as $\mathrm{SBP}>140 \mathrm{~mm} \mathrm{Hg}$ and/or DBP $>90 \mathrm{~mm} \mathrm{Hg}$ and/or receiving antihypertensive treatment. Participants with confirmed hypertension and no identified cause of secondary hypertension were diagnosed with essential hypertension.

Assessment of cognitive function. Cognitive function was measured using the 30-item Mini-Mental State Examination (MMSE), which is a global test with components of orientation, attention, calculation, language and recall. Cognitive impairment was defined as a score below 24 in the MMSE. This score has been shown to be $80-90 \%$ sensitive and $80-100 \%$ specific for a diagnosis of dementia. ${ }^{20,21}$ To decrease methodological errors and assure methodological reliability, the administrator reviewed the MMSE procedure and grading system outlined in a short booklet and video, observed a geriatrician conducting the MMSE on residents not part of the study and was supervised when conducting the MMSE on residents not part of the study. The MMSE was administered on individuals who gave consent for study participation. The individuals were categorized as follows: cognitive impairment (scores between 0 and 18), mild cognitive impairment (scores between 19 and 24) and normal (scores between 25 and 30). A diagnosis of vascular dementia was considered for individuals with dementia combined with a history or clinical evidence of stroke, and a diagnosis of non-vascular dementia was considered for individuals with dementia without a history or clinical evidence of stroke.

Assessment of covariates. Trained study personnel conducted face-to-face interviews, including standardized inquiries for self-reported medical history, medication and anthropometric measurements, as well as a standardized physical examination and a 12-lead electrocardiogram. On the basis of the prepared questionnaire for the medical record, we obtained information on age (years), gender (female/male), educational levels (illiteracy, primary school, secondary school, college or post-graduate), sleep time per day, satisfaction of sleep (very satisfied, generally satisfied or not satisfied), sleep at noon (every day, usually, occasionally or never), religion (yes or no), temperament (introversion, general or extroversion), smoking habits, alcohol consumption, tea consumption, exercise and activities of daily living (ADL). Body mass index $\left(\mathrm{kg} \mathrm{m}^{-2}\right)$ was calculated as body weight (kilograms) divided by height (meters squared). Habits of smoking, alcohol consumption, tea consumption and exercise (including former and current) were collected using a general questionnaire in which every item had two options (yes or no). ADL was measured using the MDS-ADL scale, which is composed of seven ADL items (scores ranging from 0 to 7$).^{22}$ Other variables were collected using a general questionnaire.

\section{Statistical analysis}

All statistical analyses for this study were performed with the SPSS for Windows software package, version 11.5 (SPSS Inc., Chicago, IL, USA). In the oldest-old sample, that is, the centenarians, the prevalence rate of cognitive impairment was $86.6 \%$ (including mild cognitive impairment $(27.1 \%$ ) and dementia $(59.5 \%))$; therefore, we combined mild cognitive impairment and normal cognitive function in this analysis. We compared baseline characteristics between those with and without prevalent cognitive impairment and hypertension using the $\chi^{2}$ or Fisher's exact test (in which an expected cell count was $<5$ ) for categorical variables and unpaired Student's $t$-test for continuous variables. We used analysis of variance to test for differences in cognitive function scores among the four arterial BP groups. Multiple logistic regressions were used to estimate odds ratios (ORs) and 95\% confidence intervals for hypertension as a function of increased cognitive impairment and for cognitive impairment as a function of increased hypertension. To assess the association between hypertension and cognitive impairment beyond that explained by demographic and clinical covariates, we calculated multivariate-adjusted ORs for each hypertension and cognitive impairment index. Two-sided $P$-values were considered to be statistically significant at $<0.05$.

\section{RESULTS}

Baseline characteristics and prevalence of cognitive impairment and hypertension

Among the 782 participants, mean age was 93.62 years, 76 were centenarians and 527 were women. Ninety percent of participants lived in the countryside. The mean cognitive function score for the population was 14.95 (s.d.: 5.99, range: 0-28). Only 105 volunteers had scores higher than 24 , so we defined cognitive impairment as a score lower than 19. In the oldest-old sample, the prevalence of cognitive impairment was $59.5 \%$. Of the participants with cognitive impairment, 14 had vascular dementia (3.01\%). The prevalence rate of hypertension was $57.1 \%$, including stage $1(32.9 \%)$ and stage $2(24.2 \%)$ hypertension. Of those with hypertension, $43.9 \%$ had isolated systolic hypertension and $1.9 \%$ had isolated diastolic hypertension, and only $35(7.88 \%)$ were receiving antihypertensive medication. There were 229 participants $(43.0 \%)$ who were classified as pre-hypertensive.

Participants with hypertension had a higher body mass index than those without hypertension (mean difference: $0.77, P=0.004$ ), but did not differ significantly on other covariates, including age, gender, educational levels, ADL problems, temperament, or sleeping, smoking, alcoholic, tea, exercise or religious habits (Table 1). The prevalence of cognitive impairment in women was higher than that in men $(P<0.001)$. Participants with and without cognitive impairment also showed significant differences in sleeping habits, temperament and educational levels (Table 1).

\section{Comparison of cognitive impairment prevalence and cognitive function scores between participants with and without hypertension}

There were no significant differences between participants with and without hypertension with regard to cognitive function scores $(14.95 \pm 6.01$ vs. $14.95 \pm 5.82, P=0.997)$ or with regard to the prevalence of cognitive impairment (59.42 vs. 59.52, $P=0.976$ ). Cognitive function scores are shown by BP classifications (Figure 1), and analysis of variance was used to test for differences in cognitive function scores among arterial $\mathrm{BP}$ groups $(\mathrm{F}=0, P=0.997)$.

Comparison of hypertension prevalence between participants with and without cognitive impairment

There were no significant differences between participants with and without cognitive impairment with regard to the prevalence of hypertension (56.99 vs. $57.10, P=0.976)$ or levels of arterial BP (including SBP and DBP) (139.86 \pm 22.69 vs. $140.28 \pm 23.51, P=0.799$; $73.05 \pm 12.07$ vs. $72.11 \pm 12.06, P=0.678$; for SBP and DBP, respectively) (Figure 2). Moreover, there were no significant differences in the prevalence of hypertension between participants with and without vascular dementia or between participants with and without nonvascular dementia (Table 1). We further compared levels of arterial BP between participants with vascular and non-vascular dementia $(140.04 \pm 27.32$ vs. $139.78 \pm 43.51, \quad P=0.846 ; \quad 73.11 \pm 10.13$ vs. $71.68 \pm 9.19, P=0.479$; for SBP and DBP, respectively).

\section{Multivariate-adjusted associations between cognitive impairment and hypertension}

We further assessed whether cognitive impairment was associated with increased risk of hypertension and vice versa (Table 2). After adjustment for age, gender, ADL problems, temperament, educational levels, and sleeping, smoking, alcoholic, tea, exercise and religious habits, the OR of cognitive impairment as a function of increased hypertension was $0.938(0.655,1.341)$ and the OR of hypertension as a function of increased cognitive impairment was $0.920(0.643,1.317)$ (Table 2). 
Table 1 Baseline characteristics according to arterial blood pressure and cognitive function ( $n=782$ )

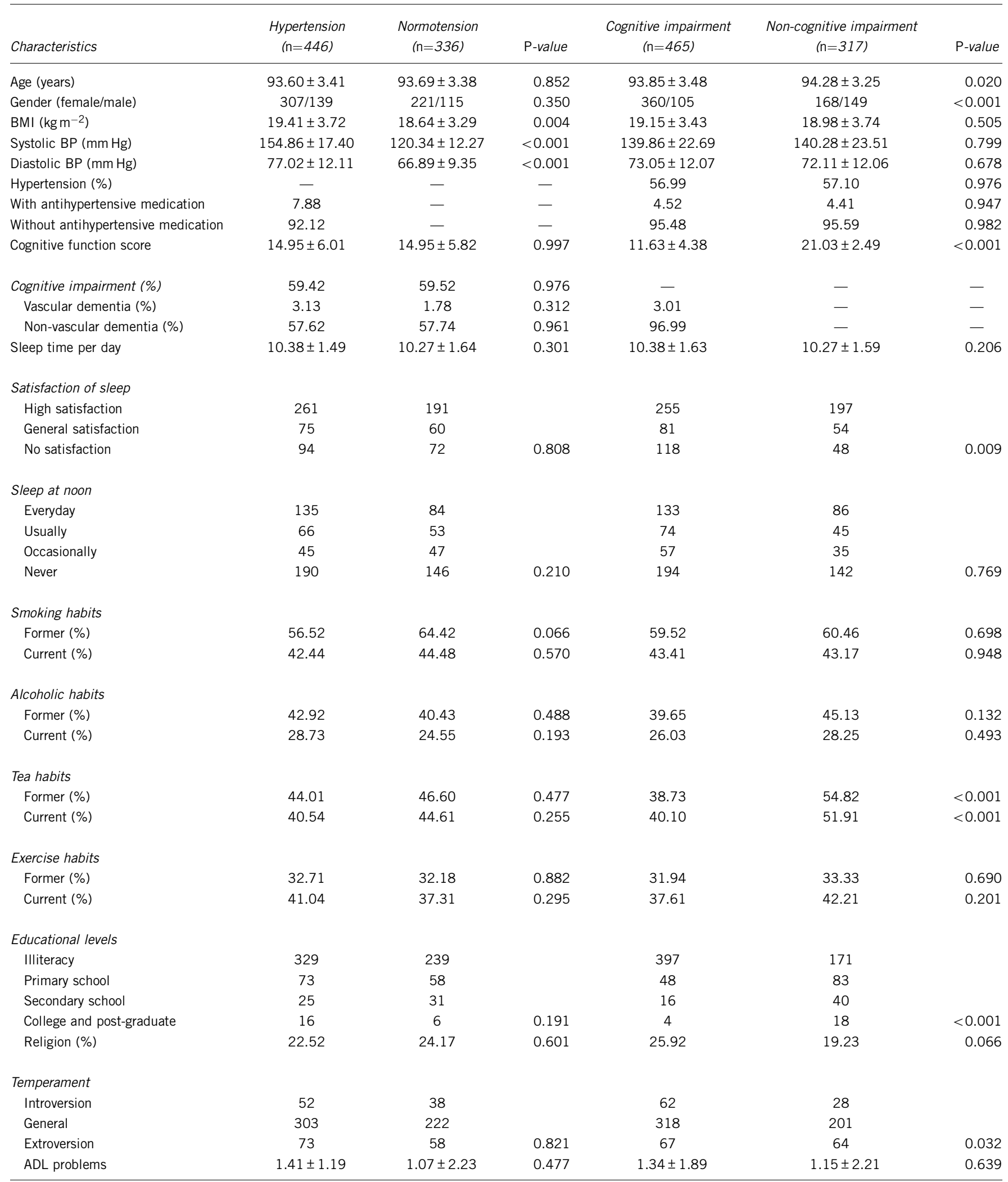

Abbreviations: ADL, activities of daily living; BMI, body mass index; BP, blood pressure.

Baseline characteristics were compared between those with and without prevalent hypertension or between those with and without prevalent depression using $\chi^{2}$ or Fisher's exact test (where an expected cell count was $<5$ ) for categorical variables and unpaired Student's $t$-test for continuous variables. In the testing, a $P$-value $<0.05$ was considered to be statistically significant. 


\section{DISCUSSION}

In this cross-sectional sample of community-dwelling Chinese nonagenarians and centenarians, we observed a high prevalence of both cognitive impairment $(59.5 \%)$ and hypertension (57.1\%). There were no significant differences in either cognitive function scores or in the prevalence of cognitive impairment between participants with and without hypertension, nor were there significant differences in either levels of arterial BP or in the prevalence of hypertension between

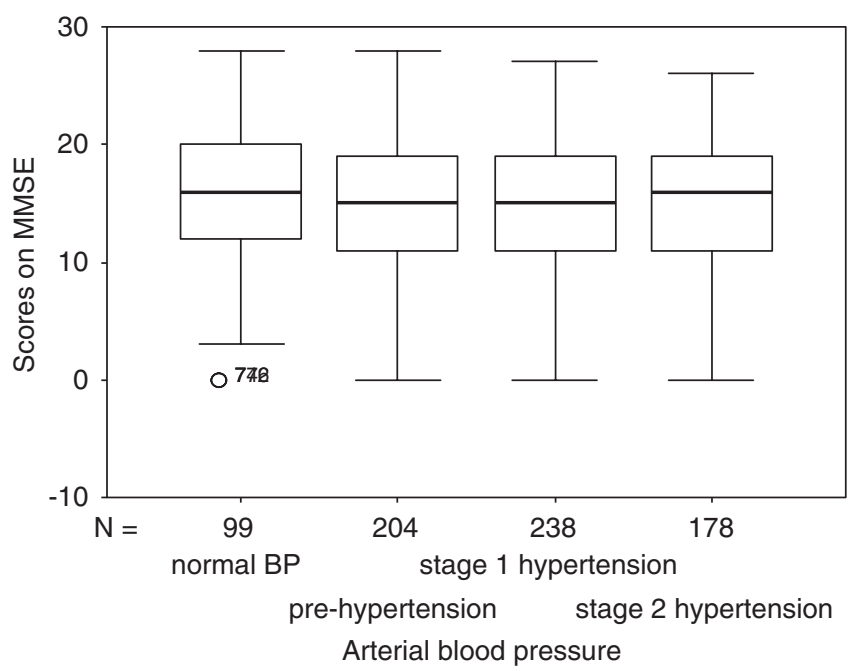

Figure 1 The analysis of variance was used to test for differences owing to depression scores among the different arterial BP groups $(F=0.000$, $P=0.997)$.

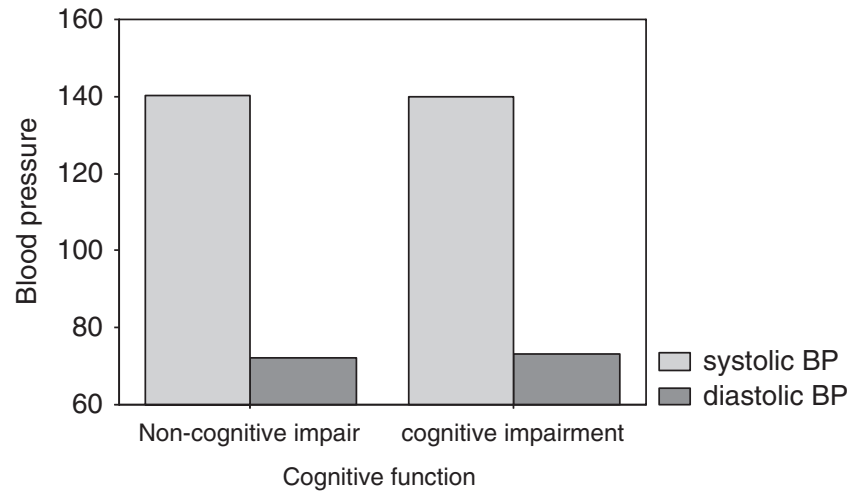

Figure 2 Unpaired Student's $t$-test was used to test for differences owing to arterial BP between participants with and without cognitive impairment. $(139.86 \pm 22.69$ vs. $140.28 \pm 23.51, P=0.799$ and $73.05 \pm 12.07$ vs. $72.11 \pm 12.06, P=0.678$, for SBP and DBP, respectively.) participants with and without cognitive impairment. Furthermore, cognitive impairment and hypertension were not risk factors for each other.

To the best of our knowledge, this was the first research that focused on the association between cognitive impairment and hypertension in nonagenarians and centenarians. This study provides useful new information in that cognitive impairment and hypertension are independent of each other among nonagenarians and centenarians. It is generally thought that hypertension and cognitive impairment are significantly and highly correlated in both younger adults and the elderly. ${ }^{23-25}$ Many earlier studies have inferred causality between hypertension and cognitive impairment in old age. Some studies have found that hypertension is a risk factor for the development of cognitive decline; on the other hand, others report that hypertension in old age is a concomitant phenomenon of already-existing cognitive impairment, rather than an independent risk factor. ${ }^{23-25}$ Our findings in this very elderly population are not consistent with studies based on people aged 65 years or older. However, participants in these earlier studies were younger and had better cognitive function and lower prevalence of hypertension than the participants in this study. Cognitive impairment and hypertension are both components of age-related deterioration. Imhof et al. ${ }^{26}$ reported that, in nonagenarians and centenarians, brain aging is characterized by the formation of neurofibrillary tangles and senile plaques, which are generally viewed as pathological changes in patients with Alzheimer's disease and dementia. This study showed that individuals with cognitive impairment were older than those without cognitive impairment; therefore, age-related deterioration was the main reason for the higher prevalence of cognitive impairment among the very elderly. ${ }^{26}$ Therefore, it is a reasonable inference that the differences between individuals with regard to cognitive function, in this study and in earlier studies, were mainly because of the different age structures of the studied groups. Further, within the nonagenarian and centenarian populations, cognitive impairment from age-related deterioration may attenuate the contribution of general risk factors for cognitive impairment, such as hypertension. On the other hand, it has been confirmed that hypertension in the elderly differs from that in younger individuals in terms of etiology, presentation, treatment and outcome. This study shows that hypertension in the oldest-old population may exhibit a different association with cognitive impairment than the association seen in the general population. Hypertension in this population was also mainly a function of age-related deterioration. Therefore, although our results did not show an association between cognitive impairment and hypertension among nonagenarians/centenarians, this is not necessarily contradictory with earlier studies; in fact, this study extends the findings of earlier studies.

This study not only provided evidence that women were more likely than men to exhibit cognitive impairment, but our findings also support those of earlier studies showing a higher risk of cognitive

Table 2 Multiple logistic regression was used to estimate the OR and $95 \% \mathrm{Cl}$ of cognitive impairment as a function of increased hypertension or hypertension as a function of increased cognitive impairment

\begin{tabular}{|c|c|c|c|c|}
\hline & \multicolumn{2}{|c|}{ Depression } & \multicolumn{2}{|c|}{ Hypertension } \\
\hline & Hypertension & Normotension & Cognitive impairment & Non-cognitive impairment \\
\hline Unadjusted OR (Cl) & $0.996(0.746,1.328)$ & 1.00 (reference) & $0.996(0.746,1.328)$ & 1.00 (reference) \\
\hline Multiple adjusted OR (Cl) & $0.920(0.643,1.317)$ & 1.00 (reference) & $0.938(0.655,1.341)$ & 1.00 (reference) \\
\hline
\end{tabular}

Abbreviations: $\mathrm{ADL}$, activities of daily living; $\mathrm{Cl}$, confidence interval; OR, odds ratio.

Adjusted for age, gender, sleep habits, smoking habits, alcoholic habits, tea habits, exercise habits, educational levels, religion habits, ADL problems and temperament. 
impairment among the very elderly. Furthermore, tea-drinking habits (former and current), satisfaction of sleep, educational level and temperament were all associated with cognitive impairment among the very elderly. This finding has implications for the prevention of cognitive impairment in the elderly; such prevention strategies may include advocating for tea use and extroverted behavior, and improving satisfaction of sleep.

Our study also has several limitations that deserve mention. First, there was a gender imbalance in our population, a common characteristic of very old populations. Of the 870 long-living individuals ( $\geqq 90$ years) in the Dujiangyan district (2005), 527 women and 255 men were included in this study. Second, we assessed cognitive function using MMSE scores, although the Diagnostic and Statistical Manual of Mental Disorders-IV is considered to be the gold standard for the diagnosis of dementia. When this cross-sectional study was conducted in 2005, assessments using the Diagnostic and Statistical Manual of Mental Disorders-IV had not been carried out; therefore, this criterion was not available for use. However, compared with the Diagnostic and Statistical Manual of Mental Disorders-IV, diagnosis of dementia by MMSE has been shown to be $80-90 \%$ sensitive and 80 $100 \%$ specific. Therefore, the diagnosis of cognitive impairment in this study was also considered to be reliable. Third, the participants in this study with both dementia and a history or clinical evidence of stroke were classified as having vascular dementia. Therefore, even individuals for whom dementia was independent of stroke may have been given a diagnosis of vascular dementia. As the prevalence of supreme cognitive impairment was high, and we did not identify the onset of cognitive impairment, we were unable to confirm the temporal relationship between stroke and dementia. As stroke is a definite risk factor for cognitive impairment, it was reasonable to assume that stroke was a cause of dementia in individuals with dementia and a history or clinical evidence of stroke. Fourth, although there may be differences in BP measurements between the sitting and recumbent positions, we did not differentiate the two. In general, the BP measurements in the sitting and recumbent positions were different, especially in the older- and oldest-aged population. Therefore, body position may be a potential confounder in BP measurements. BP in the recumbent position may be higher than that in the sitting position, and individuals with dementia were more likely to be measured in the recumbent position. Therefore, body position may increase the association of cognitive impairment with hypertension, but may not remove it. Finally, we were unable to adjust for other potential confounders, such as socioeconomic status and family history of cognitive impairment. Most (90\%) participants lived in the countryside in this study, and some had been working on a farm everyday, making physical activity a potential confounder. Therefore, this population might not be representative of the urban aged population.

In conclusion, we found no significant correlation between cognitive impairment and hypertension among nonagenarians and centenarians.

\section{CONFLICT OF INTEREST}

The authors declare no conflict of interest.
1 Evans DA. Estimated prevalence of Alzheimer's disease in the United States. Milbank Q 1990; 68: 267-289.

2 Freitag MH, Peila R, Masaki K, Petrovitch H, Ross GW, White LR, Launer LJ. Midlife pulse pressure and incidence of dementia: the Honolulu-Asia Aging Study. Stroke 2006; 37: 33-37.

3 Kivipelto M, Helkala EL, Hanninen T, Laakso MP, Hallikainen M, Alhainen K, Soininen $\mathrm{H}$, Tuomilehto J, Nissinen A. Midlife vascular risk factors and late-life mild cognitive impairment: a population-based study. Neurology 2001; 56: 1683-1689.

4 Khachaturian AS, Zandi PP, Lyketsos CG, Hayden KM, Skoog I, Norton MC, Tschanz JT, Mayer LS, Welsh-Bohmer KA, Breitner JCS, for the Cache County Study Group. Antihypertensive medication use and incident alzheimer disease: The Cache County Study. Arch Neurol 2006; 63: 686-692.

5 Nagai M, Hoshide S, Ishikawa J, Shimada K, Kario K. Ambulatory blood pressure as an independent determinant of brain atrophy and cognitive function in elderly hypertension. J Hypertens 2008; 26: 1636-1641.

6 Cherubini A, Lowenthal D, Paran E, Mecocci P, Williams L, Senin U. Hypertension and cognitive function in the elderly. Am J Ther 2007; 14: 533-554.

7 Launer LJ, Masaki K, Petrovitch H, Foley D, Havlik RJ. The association between midlife blood pressure levels and late-life cognitive function: the Honolulu-Asia Aging Study. JAMA 1995; 274: 1846-1851.

8 Boller F, Vrtunski PB, Mack JL, Kim Y. Neuropsychological correlates of hypertension. Arch Neurol 1977; 34: 701-705.

9 Farmer ME, White LR, Abbott RD, Kittner SJ, Kaplan E, Wolz MM, Brody JA, Wolf PA. Blood pressure and cognitive performance: the Framingham study. Am J Epidemiol 1987; 126: 1103-1114.

10 Cacciatore F, Abete P, Ferrara N, Paolisso G, Amato L, Canonico S, Maggi S, Varricchio $\mathrm{M}$, Rengo $\mathrm{F}$. The role of blood pressure in cognitive impairment in an elderly population. J Hypertens 1997; 15: 135-142.

11 Hebert LE, Scherr PA, Bennett DA, Bienias JL, Wilson RS, Morris MC, Evans DA. Blood pressure and late-life cognitive function change: a biracial Iongitudinal population study. Neurology 2004; 62: 2021-2024.

12 Reinprecht F, Elmstahl S, Janzon L, Andre-Petersson L. Hypertension and changes of cognitive function in 81-year-old men: a 13-year follow-up of the population study 'Men born in 1914', Sweden. J Hypertens 2003; 21: 57-66.

13 Posner HB, Tang MX, Luchsinger J, Lantigua R, Stern Y, Mayeux R. The relationship of hypertension in the elderly to $A D$, vascular dementia, and cognitive function. Neurology 2002; 58: 1175-1181.

14 Starr JM, Whalley LJ, Inch S, Shering PA. Blood pressure and cognitive function in healthy old people. J Am Geriatr Soc 1993; 41: 753-756.

15 Jama JW, Launer U, Witteman JCM, den Breeijen JH, Breteler MM, Grobbee DE, Hofman A. Dietary antioxidants and cognitive function in a population-based sample of older persons. The Rotterdam Study. Am J Epidemiol 1996; I44: 275-280.

16 Johnson LE. Vitamin disorders in the elderly. In: Morley JE, Glick Z, Rubenstein 12 (eds). Geriatric Nutrition. Raven Press: New York, 1990, 117-147.

17 van Exel E, de Craen AJ, Gussekloo J, Houx P, Bootsma-van der Wiel A, Macfarlane PW, Blauw GJ, Westendorp RG. Association between high-density lipoprotein and cognitive impairment in the oldest old. Ann Neurol 2002; 51: 716-721.

18 Rosa MO, Ana MR, Pedro A, Ana ML, Elena Q. Dietary intake and cognitive function in a group of elderly people. Int J Clin Nutr 1997; 66: 803-809.

19 Huang CQ, Dong BR, Wu HM, Zhang YL, Wu JH, Lu ZC, Flaherty JH. Association of cognitive impairment with serum lipid/lipoprotein among Chinese nonagenarians and centenarians. Dement Geriatr Cogn Disord 2009; 27: 111-116.

20 Tombaugh TN, McIntyre NJ. The mini-mental state exam: a comprehensive review. J Am Geriatr Soc 1992; 40: 922-935.

21 Langley LK. Cognitive assessment of older adults, in assessing older persons. In: Kane RL, Kane RA (eds). Oxford University Press: Oxford, UK, 2000, pp 65-128.

22 Morris JN, Fries BE, Morris SA. Scaling ADL's within the MDS. J Gerontol 1999; 4: M546-M553.

23 Qiu Cx, Winblad B, Fratiglioni L. The age-dependent relation of blood pressure to cognitive function and dementia. Lancet Neurol 2005; 4: 487-499.

24 Anson O, Paran E. Hypertension and cognitive functioning among the elderly: an overview. Am J Ther 2005; 12: 359-365.

25 Vicario A, Martinez CD, Baretto D, Diaz Casale A, Nicolosi L. Hypertension and cognitive decline: impact on executive function. J Clin Hypertens 2005; 7: 598-604.

26 Imhof A, Kövari E, von Gunten A, Gold G, Rivara CB, Herrmann FR, Hof PR, Bouras C, Giannakopoulos P. Morphological substrates of cognitive decline in nonagenarians and centenarians: a new paradigm? J Neurol Sci 2007; 257: 72-79. 